\title{
A genome-wide association study for susceptibility and infectivity of Holstein Friesian dairy cattle to digital dermatitis
}

\author{
F. Biemans, ${ }^{1,2 *}$ M. C. M. de Jong, ${ }^{1}$ and P. Bijma ${ }^{2}$ \\ ${ }^{1}$ Quantitative Veterinary Epidemiology, Wageningen University and Research, $6700 \mathrm{AH}$ Wageningen, the Netherlands \\ ${ }^{2}$ Animal Breeding and Genomics, Wageningen University and Research, $6700 \mathrm{AH}$ Wageningen, the Netherlands
}

\section{ABSTRACT}

Selection and breeding can be used to fight transmission of infectious diseases in livestock. The prevalence in a population depends on the susceptibility and infectivity of the animals. Knowledge on the genetic background of those traits would facilitate efficient selection for lower disease prevalence. We investigated the genetic background of host susceptibility and infectivity for digital dermatitis (DD), an endemic infectious claw disease in dairy cattle, with a genome-wide association study (GWAS), using either a simple linear mixed model or a generalized linear mixed model based on epidemiological theory. In total, 1,513 Holstein-Friesian cows of 12 Dutch dairy farms were scored for DD infection status and class (M0 to M4.1) every 2 wk for 11 times; 1,401 of these cows were genotyped with a $75 \mathrm{k}$ SNP chip. We performed a GWAS with a linear mixed model on 10 host disease status traits, and with a generalized linear mixed model with a complementary log-log link function (GLMM) on the probability that a cow would get infected between 2 scorings. With the GLMM, we fitted SNP effects for host susceptibility and host infectivity, while taking the variation in exposure of the susceptible cow to infectious herd mates into account. With the linear model we detected 4 suggestive SNP (false discovery rate $<0.20$ ), 2 for the fraction of observations a cow had an active lesion on chromosomes 1 and 14, one for the fraction of observations a cow had an M2 lesion on at least one claw on chromosome 1 (the same SNP as for the fraction of observations with an active lesion), and one for the fraction of observations a cow had an M4.1 lesion on at least one claw on chromosome 10. Heritability estimates ranged from 0.09 to 0.37. With the GLMM we did not detect significant nor suggestive SNP. The SNP effects on disease status analyzed with the linear model had a correlation coefficient of only 0.70 with SNP effects on susceptibility of the GLMM, indicating that both models capture partly dif-

\footnotetext{
Received October 18, 2018.

Accepted March 19, 2019.

*Corresponding author: floor.biemans@wur.nl
}

ferent effects. Because the GLMM better accounts for the epidemiological mechanisms determining individual disease status and for the distribution of the y-variable, results of the GLMM may be more reliable, despite the absence of suggestive associations. We expect that with an extended GLMM that better accounts for the full genetic variation in infectivity via the environment, the accuracy of SNP effects may increase.

Key words: genetic parameter, heritability, generalized linear mixed model, claw health, transmission

\section{INTRODUCTION}

Selection and breeding can be used to fight transmission of infectious diseases in livestock. The endemic disease digital dermatitis (DD) is a claw disorder with a genetic background (van der Waaij et al., 2005, van der Linde et al., 2010, van der Spek et al., 2013). Digital dermatitis is transmitted via the environment, and the infectious "agent" is a combination of different bacteria (Rodríguez-Lainz et al., 1996; Read and Walker, 1998; Demirkan et al., 1999; Sogstad et al., 2005; Vink et al., 2009). Digital dermatitis is prevalent in dairy cattle around the world (Argáez-Rodríguez et al., 1997; Rodriguez-Lainz et al., 1998; Wells et al., 1999; Manske et al., 2002; Holzhauer, 2006; Laven and Logue, 2006; Nielsen et al., 2009). In the Netherlands, $90.9 \%$ of the investigated herds were affected by DD in 2002-2003 (Holzhauer et al., 2006). Digital dermatitis is an important infectious disease because it affects animal welfare (Bruijnis et al., 2012) and is associated with costs for prevention and treatment (Bruijnis et al., 2010).

Infected cows have round lesions that are sometimes painful, along the coronary band of the (hind) claws (Walker et al., 1995; Read and Walker, 1998; Sogstad et al., 2005). There are 6 distinct lesion classes: skin where a lesion is macroscopically absent (M0), a small lesion of 0 to $2 \mathrm{~cm}$ (M1), a lesion of $>2 \mathrm{~cm}$ (M2), a lesion covered by a scab (M3), altered skin with dyskeratosis or surface proliferation (M4), and a small lesion in addition to altered skin (M4.1; Döpfer et al., 1997; Döpfer, 2009; Berry et al., 2012). Classes M1, M2, and M4.1 describe circumscribed, red-grayish, moist, 
painful, and prone to bleed lesions, and are sometimes referred to as the active classes (Speijers et al., 2010; Berry et al., 2012; Zinicola et al., 2015).

Genetic variants associated with DD can be detected in a genome-wide association study (GWAS) with the use of molecular markers such as SNP. Here, we use a GWAS to detect genetic variants associated with different host disease status traits for DD. We use a linear model to associate SNP with these traits. Results of previous GWAS studies on host susceptibility for DD are inconsistent. They detected significant peaks on only BTA26 (Scholey, 2011); on BTA1, 5, 8, 14, and 26 (Malchiodi et al., 2015); not at all (van der Spek et al., 2015); on BTA3, 8, and 29 (Oberbauer et al., 2016); or on BTA3 and 9 (Naderi et al., 2018).

The previous GWAS on DD focused on host disease status traits, and therefore captured effects associated with susceptibility (or resistance) only. Epidemiological models, however, assume that infectious disease transmission in a livestock population is affected by 2 sets of host traits, those affecting susceptibility and those affecting infectivity. In these models, the probability that a cow gets infected within a time interval is a function of the susceptibility of the focal cow and the total infectivity of all the infectious herd mates. When infectivity contains a heritable component, selection for lower infectivity can also be used to improve populations by selective breeding (Lipschutz-Powell et al., 2012; Anche et al., 2014; Anacleto et al., 2015). Host susceptibility and infectivity effects can be analyzed with a generalized linear mixed model with a complementary log-log link function (GLMM) that also takes into account variation in exposure of susceptible cows to infected herd mates (Anche et al., 2015; Biemans et al., 2017). Therefore, we also perform a GWAS with a GLMM to associate SNP with both host susceptibility and infectivity.

Thus, in this study we perform a GWAS with 2 different models. The first model is a linear mixed model to associate SNP with host disease status. With this model we estimate genetic parameters for 10 different DD-related host traits. The second model is a generalized linear mixed model with a complementary log-log link function, based on epidemiological theory. This model associates SNP with both host susceptibility and infectivity, and takes the variation in exposure of the susceptible cows to infectious herd mates into account.

\section{MATERIALS AND METHODS}

\section{Data}

Phenotype Data. Phenotypes were collected on 12 dairy farms in the Netherlands between November 2014 and April 2015. Two observers (author FB being one of them) visited the farms every other week for 11 times. During the visits one observer rinsed and scored the hind claws according to the method of Relun et al. (2011), and the other observer recorded the cow ID and the DD class of the cow. Six distinct classes were scored: M0, M1, M2, M3, M4, and M4.1 (Döpfer et al., 1997; Döpfer, 2009; Berry et al., 2012). Classes M1, M2, and M4.1 describe circumscribed, red-greyish, moist, painful, and prone to bleed lesions, and are referred to as the active classes (Speijers et al., 2010; Berry et al., 2012; Zinicola et al., 2015). A claw scored as M0 was classified as susceptible, and a claw scored as M1, M2, M3, M4, or M4.1 was classified as infected and infectious.

In the study period, farmers were allowed to identify and treat lesions themselves, but were not informed on the DD status of the cows by the observers. Table 1 gives an overview of some characteristics of the farms enrolled in the study. We collected cross-sectional phenotypes on 1,513 cows; 1,401 of these cows were also genotyped. The average number of scorings per cow was 8.7 because some cows were removed from, or introduced into, the herd during the study.

Genotype Data. The breeding company (CRV, Arnhem, the Netherlands) genotyped the HolsteinFriesian cows with the Eurogenomics 10K chip (EuroGenomics, Amsterdam, the Netherlands). Genotypes of cows with a call rate of $>0.85$ were eligible for imputation $(\mathrm{n}=1,401)$. Quality control was performed on the eligible data conforming to the standard procedure of the breeding company. A SNP was included only when the observed frequency deviated $<0.15$ from expected Hardy-Weinberg frequency and the minor allele frequency was $>0.025$. Furthermore, inconsistent genotypes between parents and offspring were set to missing. The SNP that passed quality control were imputed to a set of 76,438 SNP based on the Illumina BovineSNP50 chip (Illumina, San Diego, CA) and a custom chip from the breeding company, both chips had a reference population of $>1,000$ cows with genotypes. Quality control was performed on the imputed data. A SNP was included when there was no strong deviation from Hardy-Weinberg equilibrium $(P$-value $>1 \times$ $10^{-15}$ ), the missing rate was $<0.05$, and the minor allele frequency was $>2 \%$. In total, 75,904 SNP passed the quality control and were included in the final analysis.

\section{Models}

Linear Model (Disease Status Traits). We analyzed 5 disease status traits (Table 2). The first trait was infected or not (referred to as infection status: $0=$ both hind claws M0, $1=$ only one hind claw M0, $2=$ 
Table 1. Characteristics of the farms enrolled in the study

\begin{tabular}{|c|c|c|c|c|c|c|}
\hline \multirow[b]{2}{*}{ Farm } & \multirow{2}{*}{$\begin{array}{l}\text { No. of cows } \\
\text { examined }^{1}\end{array}$} & \multirow{2}{*}{$\begin{array}{l}\text { No. of cows } \\
\text { genotyped }^{1}\end{array}$} & \multirow{2}{*}{$\begin{array}{c}\text { No. of } \\
\text { observations }^{2}\end{array}$} & \multirow{2}{*}{$\begin{array}{l}\text { Average } \\
\Delta t^{3}(\mathrm{~d})\end{array}$} & \multicolumn{2}{|c|}{ Prevalence $(\mathrm{SD})^{4}$} \\
\hline & & & & & Cow level & Claw level \\
\hline $\mathrm{A}$ & 134 & 116 & 11 & 14 & $78.0(5.4)$ & $69.6(6.6)$ \\
\hline B & 105 & 101 & 11 & 14 & $56.3(7.5)$ & $46.9(7.9)$ \\
\hline $\mathrm{C}$ & 159 & 162 & 11 & 14 & $49.7(2.8)$ & $40.2(1.9)$ \\
\hline D & 118 & 116 & 11 & 14 & $57.8(5.0)$ & $49.2(5.1)$ \\
\hline $\mathrm{E}$ & 102 & 90 & 11 & 13.55 & $62.8(5.0)$ & $54.6(5.4)$ \\
\hline $\mathrm{F}$ & 133 & 112 & 10 & 15.56 & $59.2(10.0)$ & $48.7(10.4)$ \\
\hline G & 100 & 98 & 11 & 14 & $65.6(8.1)$ & $58.2(7.6)$ \\
\hline $\mathrm{H}$ & 189 & 180 & 11 & 14 & $64.9(6.2)$ & $56.7(5.8)$ \\
\hline $\mathrm{I}$ & 104 & 75 & 11 & 14 & $56.4(5.1)$ & $45.6(4.9)$ \\
\hline $\mathrm{J}$ & 88 & 88 & 11 & 14 & $65.8(10.8)$ & $58.1(10.9)$ \\
\hline $\mathrm{K}$ & 130 & 116 & 9 & 14 & $63.6(9.6)$ & $52.5(8.5)$ \\
\hline $\mathrm{L}$ & 151 & 147 & 11 & 13.90 & $70.9(7.2)$ & $62.0(7.7)$ \\
\hline Total & 1,513 & 1,401 & 129 & 14.07 & $62.6(7.5)$ & $53.9(11.0)$ \\
\hline
\end{tabular}

${ }^{1}$ Total number of different cows on a farm.

${ }^{2}$ Total number of times the milking cows on a farm were scored.

${ }^{3}$ Average number of days between observations on a farm.

${ }^{4}$ Average percentage scored as infected (class M1, M2, M3, M4, or M4.1) with the SD (M1 = a small lesion of 0-2 cm; M2 = a lesion of $>2 \mathrm{~cm} ; \mathrm{M} 3=$ a lesion covered by a scab; M4 = altered skin with dyskeratosis or surface proliferation; and M4.1 = a small lesion in addition to altered skin).

both hind claws not M0) of each cow at each observation $k(k=1$ to 11$)$. The second trait was the presence of active lesions within the study period $[0=$ no active lesions observed (class M0, M3, or M4 only), 1 = at least one active lesion observed (M1, M2, or M4.1)]. The third trait was the fraction of scorings in which a cow had at least one active lesion $\left(\right.$ fraction $_{\text {active }}$ ). We chose the fraction, rather than the number, of scorings because differences were observed between cows in the number of times they were scored. The fourth trait was the fraction of scorings a cow had a lesion of class M $i$ (fraction $_{\mathrm{M} i}$ with $i=0,1,2,3,4$, or 4.1 ). The fifth trait was the fraction of scorings both claws were scored as M0 [i.e., the fraction of observations a cow was DD free $\left(\right.$ fraction $\left.\left._{\text {free }}\right)\right]$. Table 3 illustrates how we calculated the host disease status traits from the M-class scores that a cow received.

The association of a SNP with these disease status traits was estimated with a linear model,

$$
\mathbf{y}=\mathbf{X b}+\mathbf{Z}_{a} \mathbf{a}\left(+\mathbf{Z}_{p} \mathbf{p}\right)+\mathbf{e}
$$

where $\mathbf{y}$ is a vector of observations of the trait; $\mathbf{X}$ is the incidence matrix for the fixed effects; $\mathbf{b}$ is a vector of

Table 2. Digital dermatitis disease status traits analyzed with the linear model

Trait Dependent variable

$1=$ one claw susceptible, one claw infected

$11^{1}$

$2 \quad$ Active lesion observed ${ }^{2}$

$2=$ both claws infected

$0=$ no active lesions observed

$1=$ at least one active lesion observed

3 Fraction of observations with an active lesion ${ }^{2}$

Fraction $_{\text {active }}=\frac{\text { no. of observations with active lesion on at least } 1 \text { claw }}{\text { no. of observations total }} \quad 1$

4 Fraction of observations with a lesion of class $\mathrm{M} i^{3}$

Fraction $_{\mathrm{M} i}=\frac{\text { no. of observations with class } \mathrm{M} i \text { on at least } 1 \text { claw }}{\text { no. of observations total }}$

Fraction $_{\text {free }}=\frac{\text { no. of observations with class } \mathrm{M} 0 \text { on both claws }}{}$

5 Fraction of observations with $\mathrm{M} 0^{4}$ on both claws no. of observations total

${ }^{1}$ Cows had a maximum of 11 records.

${ }^{2}$ Class M1, M2, and M4.1 are active lesions (M1 = a small lesion of 0-2 $\mathrm{cm} ; \mathrm{M} 2=\mathrm{a}$ lesion of $>2 \mathrm{~cm}$; and M4.1 = a small lesion in addition to altered skin)

${ }^{3} i=0,1,2,3,4$, or 4.1 .

${ }^{4} \mathrm{M} 0=$ skin where a lesion is macroscopically absent. 
Table 3. Example of M-class scores that a cow received at each observation, and the digital dermatitis disease status traits that were calculated from these scores ${ }^{1}$

\begin{tabular}{|c|c|c|c|c|c|c|c|c|c|c|c|c|c|c|}
\hline $\begin{array}{l}\text { Cow } \\
\text { ID }\end{array}$ & $\begin{array}{l}\text { Farm } \\
\text { ID }\end{array}$ & $\begin{array}{l}\text { Hind } \\
\text { claw }\end{array}$ & \multicolumn{11}{|c|}{ M class at observation } & $\begin{array}{l}\text { Total no. of } \\
\text { observations }\end{array}$ \\
\hline \multirow[t]{2}{*}{2047} & $\mathrm{~L}$ & Left & 4 & 4.1 & 2 & 4 & 2 & 1 & 0 & 4 & 4 & 3 & $\mathrm{NA}^{2}$ & 10 \\
\hline & & Right & 3 & 4 & 2 & 0 & 2 & 1 & 0 & 1 & 4 & 3 & NA & 10 \\
\hline
\end{tabular}

Trait

$1 \quad$ Infection status

$2 \quad$ Active lesion observed $=1$

$3 \quad$ Fraction $_{\text {active }}=5 / 10=0.50$

$4 \quad$ Fraction $_{\mathrm{M} 0}=2 / 10=0.20$

Fraction $_{\mathrm{M} 1}=2 / 10=0.20$

Fraction $_{\mathrm{M} 2}=2 / 10=0.20$

Fraction $_{\mathrm{M} 3}=2 / 10=0.20$

Fraction $_{\mathrm{M} 4}=5 / 10=0.50$

Fraction $_{\mathrm{M} 4.1}=1 / 10=0.10$

$5 \quad$ Fraction $_{\text {free }}=1 / 10=0.10$

${ }^{1}{ }_{\text {Fraction }}$ active $=$ fraction of scorings in which a cow had at least one active lesion; fraction $_{\text {free }}=$ fraction of observations in which a cow was digital dermatitis free. Other fractions: skin where a lesion is macroscopically absent (M0), a small lesion of $0-2 \mathrm{~cm}$ (M1), a lesion of $>2 \mathrm{~cm}$ (M2), a lesion covered by a scab (M3), altered skin with dyskeratosis or surface proliferation (M4), and a small lesion in addition to altered skin (M4.1).

${ }^{2} \mathrm{NA}$ means that a cow was not scored at this observation.

the fixed effects, including SNP-genotype (0, 1, or 2), farm (A to L), and parity (1, 2, or $>2$ ), months in milk was not significant and therefore was not included as a fixed effect; $\mathbf{Z}_{a}$ is the incidence matrix for the additive genetic effects; and $\mathbf{a}$ is a vector of additive genetic effects, with $\mathbf{a} \sim N\left(\mathbf{0}, \mathbf{G} \sigma_{a}^{2}\right)$, where $\mathbf{G}$ is the additive genomic relationship matrix of the 1,401 genotyped cows (VanRaden, 2008, method 1) and $\sigma_{a}^{2}$ is the additive genetic variance; e is a vector of residual effects. For the trait infection status, we also included fixed effects for the scoring round (1 to 11) and the interaction between farm and the scoring round; and a random permanent cow effect $\mathbf{p}$ with incidence matrix $\mathbf{Z}_{p}$, to account for repeated observations on a cow. (Though a linear model is equivalent to a generalized linear model with a unity link function, we use the term "linear model" because this term is commonly used).

Generalized Linear Mixed Model (Susceptibility and Infectivity). The association of a SNP with host susceptibility and infectivity for DD was estimated with a GLMM, where the y-variable was an infection event, rather than a disease status trait. In contrast to the linear model, this GLMM is founded in epidemiological principles. With the GLMM, we took the variation in exposure of a susceptible cow to the infectious herd mates and the genetic variation in infectivity of those herd mates into account. To develop the GLMM, we need to find the probability that a susceptible cow gets infected within a scoring interval. We first present an epidemiological model, then derive the infection probability from this model, and finally present the resulting GLMM. We build on work of Velthuis et al. (2003), Lipschutz-Powell et al. (2014), Anche et al. (2015), and Biemans et al. (2017).

With the GLMM we analyzed whether susceptible claws of a cow became infected within a scoring interval. A susceptible cow had 1 or 2 susceptible claws at the start of an interval ( $\mathrm{F}=1$ or 2$)$, that were 0,1 , or 2 cases by the end of the interval $(\mathrm{C}=0,1$, or 2$)$. The dependent variable was the number of cases over the number of susceptible claws, C/F. The data set for the GLMM consisted of 6,099 scorings of cows that had at least one susceptible claw at the start of an interval (a total number of 1,513 different cows were repeatedly scored in the study).

First, we modeled DD transmission with a stochastic compartmental susceptible-infected-susceptible model with an environment route E (Figure 1; see e.g., Bravo de Rueda et al., 2015).

In this model, infection of a susceptible claw occurs randomly with a probability per unit of time depending on the model parameters, the number of infectious claws in the herd, and the infection pressure coming from the environment. The expected rate with which susceptible claws get infected is $\beta S \frac{E+I}{N}$, where $\beta$ is the transmission rate parameter, $S$ is the number of susceptible claws, $I$ is the number of infectious claws, and $N$ is the total number of claws in a group (twice the number of cows), so that $S+I=N$. The infectious claws deposit infectious material in the environment, and susceptible claws can get infected via the environment. The infection pressure coming from the environ- 

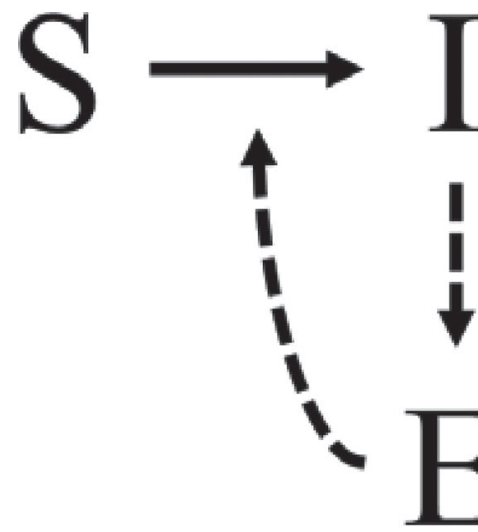

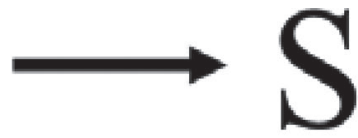

igure 1. Susceptible-infectious-susceptible model with an environment route, with the number of susceptible feet $(\mathrm{S})$, the number of infected feet (I), and the infectious pressure coming from an environmental reservoir (E).

ment, $E$, was expressed in "currently infected claw equivalents," so that $E+I$ represents the total infection pressure expressed as the equivalent number of currently infected claws. The transmission rate parameter $\beta$ is a population-specific parameter that contains information on the contact rate and the transmission probability between cows (Roberts and Heesterbeek, 1993).

Second, to find the probability that a specific cow in a specific scoring interval became infected, we need to model genetic (SNP) variation in susceptibility and infectivity between cows. Thus we considered the pairwise transmission rate parameter $\beta_{i j}$ between a susceptible claw of (focal) cow $i$ and an infectious claw of its herd mate $j$. The transmission rate parameter $\beta_{i j}$ from a single infectious claw of cow $j$ with infectivity $\varphi_{j}$ to one susceptible claw of cow $i$ with susceptibility $\gamma_{i}$ was

$$
\beta_{i j}=c \gamma_{i} \varphi_{j},
$$

where $c$ is the average contact rate. In this expression, average susceptibility and infectivity are defined to be one, $\bar{\gamma}=\bar{\varphi}=1$, so that $c$ represents the transmission rate parameter for a typical pair of cows $i$ and $j$. In other words, $c$ refers to an effective contact rate, not to a physical contact rate.

When a claw of cow $i$ is exposed to all infectious claws, the expected infection rate for this claw depends on the susceptibility of cow $i$, on the number of infectious claws in the herd, and on their average infectivity. The total infection rate equals the sum of the rates due to each infectious claw,

$$
\text { infection } \operatorname{rate}_{i}=c \gamma_{i} \frac{\sum_{j} \varphi_{j}}{I_{g}} \frac{E+I_{t o t}}{N} .
$$

The $\sum_{j} \varphi_{j} / I_{g}$ is the average infectivity of the claws of genotyped infectious cows. Because not all cows were genotyped, we distinguished between the number of infectious claws of genotyped cows $\left(I_{g}\right)$ and the total number of infectious claws $\left(I_{t o t}\right)$. Genetic differences in infectivity could be estimated for genotyped cows only. To account for the infectivity of nongenotyped cows, we included all infectious cows in the last term of equation [2].

The probability that a susceptible claw became infected within an interval followed from assuming a Poisson process within interval $\Delta t$, and is the probability of a nonzero outcome from a Poisson distribution. This probability follows from equation [2] as

$$
P_{i}(t)=1-e^{-c \gamma_{i} \frac{\sum_{\rho_{j}} \varphi_{j}}{I_{g}(t)} \frac{E(t)+I_{t o t}(t)}{N(t)} \Delta t},
$$

where $P_{i}(t)$ is the probability that a susceptible claw of cow $i$ is a case (became infected) in interval $\Delta t$. Thus, for cow $i$, the number of cases within the interval, $C_{i}(t)$ $=0,1$, or 2 , follows a binomial distribution, with binomial total $F_{i}(t)=1$ or 2 , and the probability given by equation [3]. $E(t)$ is the infection pressure coming from the environment at time $t . N(t)$ is the total number of claws in a group (twice the number of cows) at time $t$.

Finally, we present the resulting GLMM. Because the probability of being a case follows a Poisson process, the complementary log-log (cloglog) is the appropriate link function to connect the explanatory variables to the expected probability to be infected (equation [3]),

$$
\begin{aligned}
& \operatorname{clog} \log \left[P_{i}(t)\right]= \\
& \log (c)+\log \left(\gamma_{i}\right)+\log \left(\frac{\sum_{j} \varphi_{j}}{I_{g}(t)}\right)+\log \left(\frac{E(t)+I_{t o t}(t)}{N(t)} \Delta t\right) .
\end{aligned}
$$

However, equation [4] is not linear in the logarithm of infectivity. To solve this issue, we moved the number of infectious claws of cows that were genotyped to the offset, and approximated $\log \left(\frac{\sum_{j} \varphi_{j}}{I_{g}(t)}\right)$ by $\frac{1}{I_{g}(t)} \sum_{j} \log \left(\varphi_{j}\right)$, which is equivalent to approximating the $\log$ of an arithmetic mean by the log of the corresponding geometric mean (Anche et al., 2015; Biemans et al., 2017), so that

$\operatorname{clog} \log \left[P_{i}(t)\right] \approx$

$\log (c)+\log \left(\gamma_{i}\right)+\frac{1}{I_{g}(t)} \sum_{j} \log \left(\varphi_{j}\right)+\log \left(\frac{E(t)+I_{\text {tot }}(t)}{N(t)} \Delta t\right)$. 
The last term in equation [5], $\log \left(\frac{E(t)+I_{t o t}(t)}{N(t)} \Delta t\right)$ is an offset (i.e., a known effect treated as an "explanatory variable" with a fixed regression coefficient of 1 ). The offset accounts for the fraction of infectious claws at $t\left[I_{t o t}(t) / N(t)\right]$, the length of the interval $(\Delta t)$, and the infection pressure coming from the environment at $t[E(t) / N(t)]$.

Details of the calculation of the infection pressure coming from the environment are described in Biemans et al. (2018). In short, claws that were infectious at an earlier stage could still (partly) contribute to the environmental reservoir at $t[E(t)]$. The contribution was assumed to decrease each interval $\Delta t$ with factor $\lambda$, which may be interpreted as a survival rate of the pathogen in the environment. For DD this survival rate was estimated to be 0.9 (Biemans et al., 2018). Therefore, the infection pressure via the environment of a single claw that was infectious at time $t$, equaled 0.9 at $t+1,0.9^{2}$ at $t+2,0.9^{3}$ at $t+3$, and so on. Hence, values for $E(t)$ were calculated as

$$
E(t)=0.9\left[E(t-1)+I_{t o t}(t-1)\right],
$$

which is the contribution of the claws that were infectious before $t$, to the environmental reservoir at $t$. For the first interval, equation [6] requires information on the number of infectious claws before observations started. We estimated this number with linear regression within herd on the number of infected claws over time, where $t=1$ represents the first observation. The intercept of the model $\left[I_{t o t}(t=0)\right]$ was used as the average number of infectious claws before observations started, and the value for the environmental reservoir before observations started was estimated as $E(t=0)=\frac{0.9}{1-0.9} I_{t o t}(t=0)$ (Biemans et al., 2018).

In the final GLMM we included fixed effects for farm, interval, parity, months in milk, a SNP effect for susceptibility, and a SNP effect for infectivity; and random effects for the farm by interval interaction, cow, a genetic effect for susceptibility, and a genetic effect for infectivity,

$$
\begin{aligned}
& \operatorname{clog} \log \left[P_{i j k l m}(t)\right]=c_{0}+\text { Farm }_{k}+\text { Interval }_{m}+\text { Parity }_{l} \\
& +c_{1} \text { MIM }+c_{2} S N P_{S U S, i}+c_{3} S_{N P_{I N F, \bar{j}}}+\text { Farm }_{k} \times \text { Interval }_{m} \\
& + \text { Animal }_{i}+\log \left(\gamma_{i}\right)+\frac{1}{I_{g}(t)} \sum_{j} \log \left(\varphi_{j}\right) \\
& +\log \left(\frac{E(t)+I_{\text {tot }}(t)}{N(t)} \Delta t\right) .
\end{aligned}
$$

Here, $P_{i j k l m}(t)$ is the expectation of the number of cases over the number of susceptible claws of cow $i$ within $\Delta t$, $E\left(C_{i}(t) / F_{i}(t)\right)$. The $c_{0}$ is the intercept; Farm $_{k}$ is a fixed farm effect $(k=\mathrm{A}$ to $\mathrm{L})$; Interval ${ }_{m}$ is a fixed effect for the scoring interval $(m=1$ to 10$)$; Parity $_{l}$ is a fixed effect for parity $(l=1,2$, or $>2) ; c_{1}$ is the fixed regression coefficient for months in milk (MIM is a discrete variable from 1 to 12$) ; c_{2}$ is the fixed regression coefficient for susceptibility, where $S N P_{S U S, i}$ represents the number of reference alleles of the susceptible cow $i$, and takes values 0,1 , or 2 ; and $c_{3}$ is the fixed regression coefficient for infectivity, where $S N P_{I N F, \bar{j}}$ represents the average number of reference alleles of the infectious claws on a farm and takes real values between 0 and 2 (Biemans et al., 2017). The random effects were the interaction between farm and interval $\left(\mathrm{Farm}_{k} \times\right.$ Inter$v a l_{m}$ ), a nongenetic permanent cow effect to account for repeatedly scored cows $\left(\right.$ Animal $\left._{i}\right)$, an additive polygenic effect for susceptibility of cow $i$

$$
\left[\log \left(\gamma_{i}\right) \text { with } \log (\gamma) \sim N\left(\mathbf{0}, \mathbf{G} \sigma_{a}^{2}\right)\right],
$$

where $\mathbf{G}$ is the genomic relationships matrix among cows), and an additive polygenic effect for infectivity of the infectious group mates $j$ of cow $i$

$$
\left[\sum_{j} \log \left(\varphi_{j}\right), \text { with } \log (\varphi) \sim N\left(\mathbf{0}, \mathbf{G} \sigma_{a}^{2}\right)\right] .
$$

The last term in model 2 is the offset.

\section{Analyses}

We fitted the linear model (model 1) for the trait infection status (Table 2, trait 1) with ASReml v4.1.0 (Gilmour et al., 2015). The G-matrix used in ASReml was computed using method 1 of VanRaden (2008) with the calc_grm software (Calus and Vandenplas, 2016). We fitted the linear models for the other disease status traits with GCTA (Yang et al., 2011) for reasons of computing time. We fitted the generalized linear mixed model (model 2) with ASReml v4.1.0 (Gilmour et al., 2015). The SNP were fitted individually in succession, starting with the first SNP on BTA1, following the genome, and ending with the last SNP on BTA29.

The significance threshold for both models was adjusted for multiple testing, using the function qvalue of the package qvalue (Storey and Tibshirani, 2003) in $\mathrm{R}$ v3.4.0 (R Core Team, 2017) to obtain the false discovery rate (FDR). If FDR $\leq 0.20$, the association was called suggestive, and if FDR $\leq 0.05$ the association was called significant (van der Spek et al., 2015). Manhat- 
tan plots and quantile-quantile plots were created with R-package qqman (Turner, 2014). Phenotypic correlations between traits were calculated in ASReml v4.1.0.

\section{RESULTS}

\section{Trait Comparison}

Table 4 shows the mean and standard deviation of all traits and the total number of phenotypes in the data set. Most traits have one phenotype per cow $(1,401$ in total) except for infection status and $\mathrm{C} / \mathrm{F}$, which include repeated scorings on cows.

Table 5 shows the phenotypic correlations between the traits analyzed with the linear model. Mean infection status had a high phenotypic correlation with fraction $_{\mathrm{M} 0}(-0.96)$, fraction ${ }_{\mathrm{M} 4}(0.93)$, and fraction ${ }_{\text {free }}$ $(-0.95)$. Furthermore, a high correlation was observed

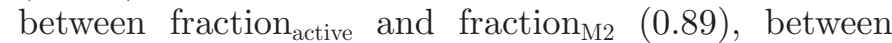
fraction $_{\mathrm{M} 4}$ and fraction $_{\text {free }}(-0.95)$, between fraction M0 $_{\mathrm{M}}$ and fraction ${ }_{\text {free }}(0.83)$, and between fraction $_{\mathrm{M} 0}$ and frac$\operatorname{tion}_{\mathrm{M} 4}(0.83)$.

\section{Fixed Effects}

In the linear model, the fixed effects for farm, scoring round, the interaction between farm and scoring round, and parity were significant $(P<0.05)$. The probability that a cow was infected with DD increased with scoring round and parity (Table 6).

In the GLMM, the farm effect was not significant. This was probably because the main difference between farms was the number of the infectious cows and the infectivity in the environment, which were accounted for by the offset. The fixed effects for interval, parity, and months in milk were significant $(P<0.05)$. The probability of getting infected with DD increased for the first 6 intervals and stabilized thereafter. The transmission rate parameter increased with increasing parity and was $21 \%$ higher for parity 2 cows compared with parity 1 cows, and $69 \%$ higher in parity $>2$ cows compared with parity 1 cows. For months in milk, the transmission rate parameter decreased with $4 \%$ per month in milk. Higher-order effects of months in milk were not significant.

\section{SNP Effects}

For all traits, the quantile-quantile plots and the allele frequency and estimated SNP effects of the suggestive SNP are in Appendix Figure A1). The quantilequantile plots show marginal inflation of $P$-values from
Table 4. Mean trait value with SD and total number of observations in the data set

\begin{tabular}{lccc}
\hline Item $^{1}$ & Mean & SD & $\begin{array}{c}\text { No. of } \\
\text { observations }\end{array}$ \\
\hline Infection status & 1.08 & 0.90 & 12,195 \\
Active lesions observed & 0.32 & 0.47 & 1,401 \\
Fraction $_{\text {active }}$ & 0.09 & 0.18 & 1,401 \\
Fraction $_{\mathrm{M} 0}$ & 0.57 & 0.41 & 1,401 \\
Fraction $_{\mathrm{M} 1}$ & 0.01 & 0.05 & 1,401 \\
Fraction $_{\mathrm{M} 2}$ & 0.06 & 0.14 & 1,401 \\
Fraction $_{\mathrm{M} 3}$ & 0.02 & 0.07 & 1,401 \\
Fraction $_{\mathrm{M} 4}$ & 0.56 & 0.38 & 1,401 \\
Fraction $_{\mathrm{M} 4.1}$ & 0.02 & 0.07 & 1,401 \\
Fraction $_{\text {free }}$ & 0.39 & 0.40 & 1,401 \\
$\mathrm{C}^{2}$ & 0.21 & 0.38 & 6,099 \\
\hline
\end{tabular}

${ }^{1}$ Fraction $_{\text {active }}=$ fraction of scorings in which a cow had at least one active lesion; fraction free $=$ fraction of observations in which a cow was digital dermatitis free. Other fractions: skin where a lesion is macroscopically absent (M0), a small lesion of $0-2 \mathrm{~cm}$ (M1), a lesion of $>2$ cm (M2), a lesion covered by a scab (M3), altered skin with dyskeratosis or surface proliferation (M4), and a small lesion in addition to altered skin (M4.1).

${ }^{2}$ Cases $(\mathrm{C})$ over the number of susceptible feet $(\mathrm{F})$; dependent variable in the generalized linear mixed model.

the linear model, whereas $P$-values from the GLMM show no inflation at all.

Linear Model (Susceptibility). For trait infection status, neither significant nor suggestive SNP were observed.

The top panel of Figure 2 shows the Manhattan plot for the presence of an active lesion. Neither significant nor suggestive SNP were observed. The bottom panel of Figure 2 shows the Manhattan plot for fraction ${ }_{\text {active }}$. No significant SNP were observed, but one suggestive SNP was observed on BTA1 and one on BTA14. Details on suggestive SNP are in Table 7.

Figure 3 shows the Manhattan plots for the fraction of scorings a cow had a class M $i$ lesion. No significant SNP were present, but 2 suggestive SNP were observed. Fraction $_{\mathrm{M} 2}$ had one suggestive SNP on BTA1; this is the same SNP as found on BTA1 for the trait fractio$\mathrm{n}_{\text {active. }}$. Fraction M $_{4.1}$ had one suggestive SNP on BTA10.

For the fraction of scoring a cow was DD free [i.e., had an M0 score on both hind claws (fraction free $_{\text {f }}$ ), neither significant nor suggestive SNP were observed. All suggestive SNP explained only a small part of the phenotypic variance (Table 7). Heritability estimates ranged from 0.093 (fraction $_{\mathrm{m} 1}$ ) to 0.367 (fraction $_{\text {free }}$ ), with small standard errors (Table 8).

Generalized Linear Mixed Model (Susceptibility and Infectivity). Figure 4 shows the Manhattan plots for susceptibility and infectivity. No significant and no suggestive SNP were observed. The $\log _{10} P$ values ranged from 0 to 5.11 for susceptibility, and from 
0 to 4.35 for infectivity. Overall, the standard errors were large, particularly for infectivity.

\section{Model Comparison}

Table 9 shows the correlations between estimated SNP effects for all traits. All traits were analyzed with the linear model (model 1) except for susceptibility and infectivity, which were analyzed with the GLMM (model 2). Infection status had a high correlation with

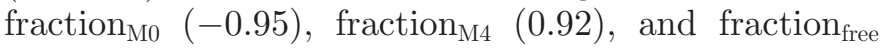
$(-0.95)$. Fraction ${ }_{\mathrm{M} 4}$ had a high negative correlation with fraction free $(-0.95)$ and with fraction ${ }_{\mathrm{M} 0}(-0.84)$. In contrast, fraction ${ }_{\text {free }}$ had a high positive correlation with fraction $_{\mathrm{M} 0}(0.84)$. Fraction ${ }_{\text {active }}$ had a moderate correlation with fraction $_{\mathrm{M} 1}(0.42)$, fraction ${ }_{\mathrm{M} 4.1}(0.48)$, and fraction free $(-0.42)$, whereas it had a high correlation with fraction M2 $(0.89)$. Susceptibility analyzed with the GLMM had a high correlation with infection status (0.70), fraction M $0(-0.63)$, fraction M $4(0.71)$, and fraction $_{\text {free }}(-0.70)$ analyzed with the linear model.

Table 6. Fixed effects estimates and SE from the linear model on infection status

\begin{tabular}{|c|c|c|c|}
\hline Fixed effect & Coefficient $^{1}$ & $\mathrm{SE}^{1}$ & $\begin{array}{c}\text { No. of } \\
\text { infectious } \\
\text { claws }^{2}\end{array}$ \\
\hline Intercept & 1.374 & 0.102 & 1.055 \\
\hline \multicolumn{4}{|l|}{ Farm } \\
\hline $\mathrm{A}$ & 0.000 & 0.000 & 1.336 \\
\hline B & -0.483 & 0.132 & 0.852 \\
\hline $\mathrm{C}$ & -0.346 & 0.116 & 0.990 \\
\hline $\mathrm{D}$ & -0.373 & 0.126 & 0.963 \\
\hline $\mathrm{E}$ & -0.310 & 0.133 & 1.026 \\
\hline $\mathrm{F}$ & -0.464 & 0.128 & 0.872 \\
\hline $\mathrm{G}$ & -0.339 & 0.127 & 0.996 \\
\hline $\mathrm{H}$ & -0.017 & 0.133 & 1.319 \\
\hline I & -0.158 & 0.149 & 1.178 \\
\hline J & -0.319 & 0.147 & 1.016 \\
\hline $\mathrm{K}$ & -0.419 & 0.129 & 0.917 \\
\hline $\mathrm{L}$ & -0.136 & 0.117 & 1.200 \\
\hline \multicolumn{4}{|c|}{ Scoring round } \\
\hline 1 & 0.000 & 0.000 & 0.910 \\
\hline 2 & 0.082 & 0.076 & 0.992 \\
\hline 3 & 0.079 & 0.077 & 0.988 \\
\hline 4 & -0.073 & 0.076 & 0.837 \\
\hline 5 & 0.157 & 0.077 & 1.067 \\
\hline 6 & 0.287 & 0.076 & 1.197 \\
\hline 7 & 0.085 & 0.075 & 0.995 \\
\hline 8 & 0.259 & 0.076 & 1.169 \\
\hline 9 & 0.148 & 0.076 & 1.057 \\
\hline 10 & 0.307 & 0.076 & 1.217 \\
\hline 11 & 0.271 & 0.077 & 1.180 \\
\hline \multicolumn{4}{|l|}{ Parity } \\
\hline 1 & -0.395 & 0.043 & 0.822 \\
\hline 2 & -0.157 & 0.040 & 1.082 \\
\hline$>2$ & 0.000 & 0.00 & 1.239 \\
\hline
\end{tabular}

${ }^{1}$ Estimates were averaged over 75,904 analyses (one for each SNP). ${ }^{2}$ For example, for farm, number of infectious claws for a cow on that farm in an average round effect with an average parity effect, similar for scoring round and parity. 

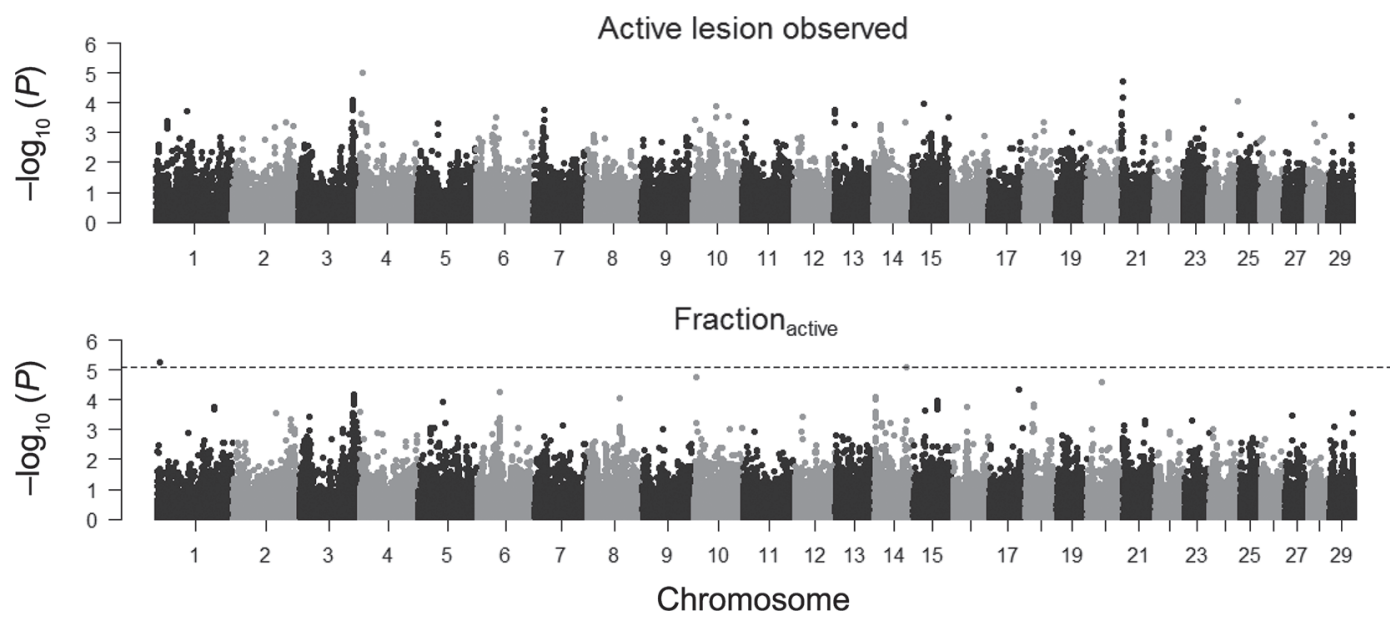

Figure 2. Genome-wide association study for presence of an active digital dermatitis lesion within the study period (top panel) and fraction of observations a cow had an active lesion (bottom panel); $\log _{10} P$-value per SNP against position on the chromosome in base pairs. The false discovery rate was set at 0.20 for suggestive SNP (dashed line). Fraction active $=$ fraction of scorings in which a cow had at least one active lesion.

Infectivity had a low correlation (between -0.14 and 0.12 ) with all traits.

\section{DISCUSSION}

We investigated the genetic background of DD with 2 models and data on 1,401 cows. In contrast to most other studies, we collected longitudinal data rather than cross-sectional data. This allowed us to not only investigate disease status traits, but also infection events. First, we used a linear model for different host disease status traits. No significant SNP (FDR $<0.05)$ were observed, but 4 suggestive SNP $($ FDR $<0.20)$ were observed for 3 traits (i.e., fraction active $_{\text {, fraction of scor- }}$ ings as M2 on at least one claw, and fraction of scorings as M4.1 on at least one claw). Second, we used a generalized linear mixed model on infection events, fitting host SNP effects for susceptibility and infectivity, while taking variation in exposure of the susceptible herd mates into account. Neither significant nor suggestive SNP were observed in this analysis. The estimated SNP effects for the trait infection status (linear model) had a correlation of 0.70 with the SNP effects for susceptibility (GLMM). Heritability estimates ranged from 0.09 for fraction $_{\mathrm{m} 1}$ to 0.37 for fraction free $_{\text {. }}$.

\section{Linear Model (Disease Status Traits)}

We analyzed, among others, the fraction of observations a cow was scored with a certain lesion class. We chose the fraction, rather than the number, of observations a cow presented a certain lesion as the dependent variable to account for the number of times a cow was scored. Scorings where a cow had the same lesions on both claws at the same observation were only counted once. This way of counting classes works well for infected claws (i.e., M1, M2, M3, M4, or M4.1) because a cow scored with one of these classes is indeed infected at that observation. However, for the class M0, a cow with one M0 claw and one infectious class claw was counted as susceptible, whereas in reality the cow is both susceptible and infected. Hence, the fraction ${ }_{\mathrm{M} 0}$ is actually the fraction of scorings a cow was not infected on at least one claw. Thus, this trait does not directly

Table 7. Details on suggestive SNP

\begin{tabular}{lrrcccr}
\hline Trait $^{1}$ & BTA & \multicolumn{1}{c}{ bp } & MAF & SNP effect & SE SNP effect & \% Variance explained \\
\hline Fraction $_{\text {active }}$ & 1 & $5,629,481$ & 0.067 & 0.0612 & 0.0134 & 1.46 \\
& 14 & $67,961,226$ & 0.030 & 0.0878 & 0.0195 & 1.41 \\
Fraction $_{\mathrm{M} 2}$ & 1 & $5,629,481$ & 0.067 & 0.0522 & 0.0110 & 1.56 \\
Fraction $_{\mathrm{M} 4.1}$ & 10 & $12,645,355$ & 0.029 & 0.0344 & 0.0074 & 1.54 \\
\hline
\end{tabular}

${ }^{1}$ Fraction $_{\text {active }}=$ fraction of scorings in which a cow had at least one active lesion; fraction ${ }_{\mathrm{M} 2}=$ fraction of scorings in which a cow had a lesion of $>2 \mathrm{~cm}$ (M2); fraction ${ }_{\mathrm{M} 4.1}=$ fraction of scorings in which a cow had a small lesion in addition to altered skin (M4.1).

${ }^{2} \mathrm{MAF}=$ minor allele frequency. 

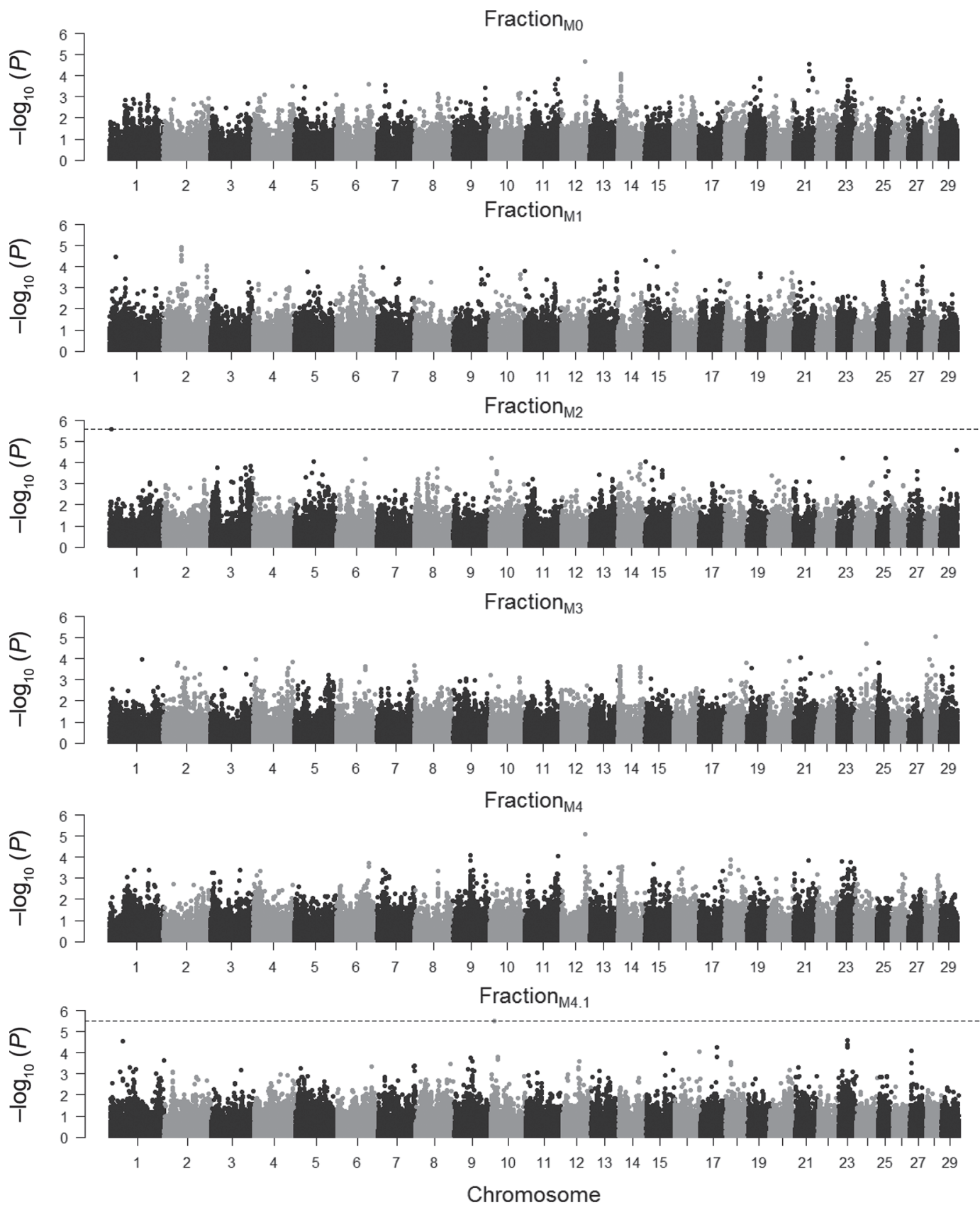

Figure 3. Genome-wide association study for fraction of observations a cow had a digital dermatitis score of M0 (skin where a lesion is macroscopically absent), M1 (a small lesion of $0-2 \mathrm{~cm}$ ), M2 (a lesion of $>2 \mathrm{~cm}$ ), M3 (a lesion covered by a scab), M4 (altered skin with dyskeratosis or surface proliferation), and M4.1 (a small lesion in addition to altered skin) on at least one $\operatorname{claw} \log _{10} P$-value per SNP against position on the chromosome in base pairs. The false discovery rate was set at 0.20 for suggestive SNP (dashed line).

reflect the susceptibility to DD. We therefore also analyzed the fraction of scoring a cow was DD free (i.e., M0 score on both hind claws).

Phenotypes and SNP effects were highly correlated for fraction $_{\mathrm{M} 0}$, fraction f , and fraction free $_{\text {. These traits }}$ have a strong relation because claws in this study were either M0, thus susceptible, or M4 the majority of the time they were infected (Biemans et al., 2018). Furthermore, a SNP on BTA1 associated with fraction active was $_{\text {a }}$ also associated with fraction ${ }_{\mathrm{M} 2}$, with similar estimates. This SNP was not found in previous GWAS on DD.

No significant SNP were associated with DD and the suggestive SNP were on different chromosomes than reported in previous studies (Scholey, 2011; Malchiodi 

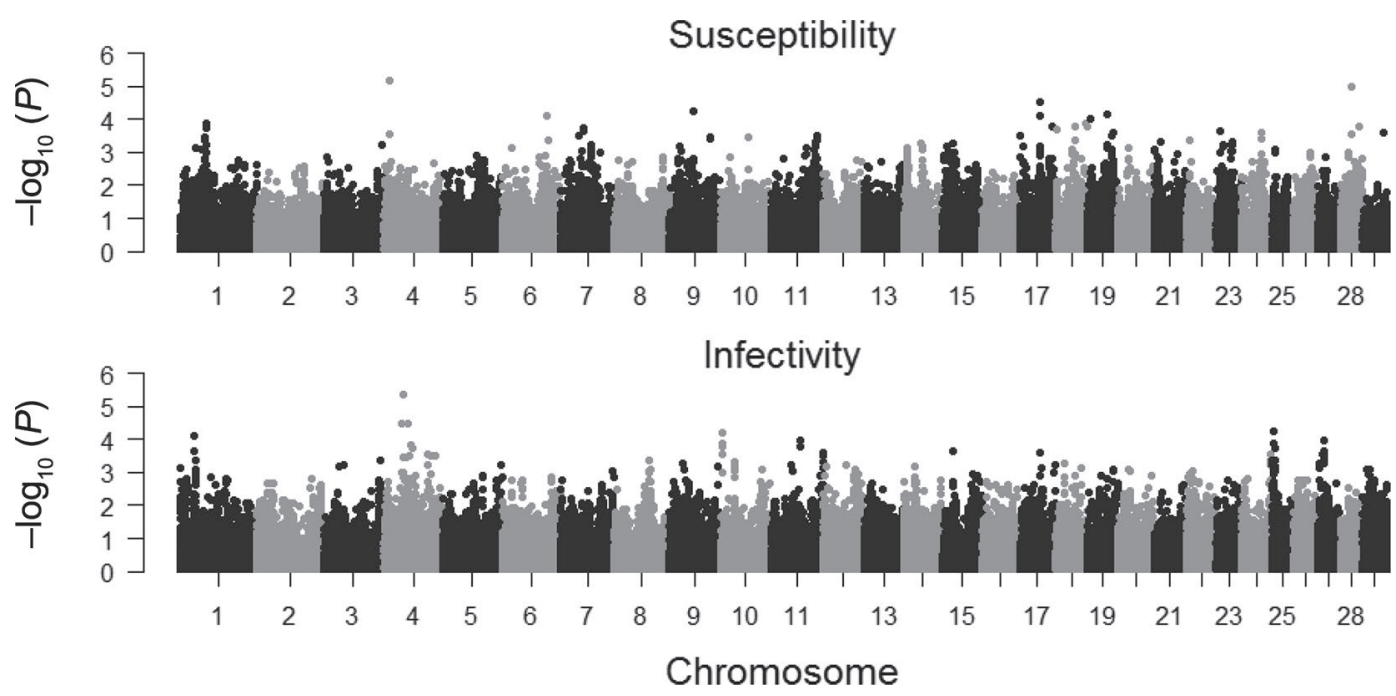

Figure 4. Genome-wide association study on host susceptibility and host infectivity for digital dermatitis: $\log _{10} P$-value per SNP against position on the chromosome in base pairs.

et al., 2015; Oberbauer et al., 2016; Naderi et al., 2018). Furthermore, another study did not find any association (significant or suggestive) with DD at all (van der Spek et al., 2015). It is possible that this difference between studies is not because of the methods or models used, but because there are no major QTL contributing to the disease.

\section{Generalized Linear Mixed Model (Susceptibility and Infectivity)}

We analyzed the probability that a cow would get infected in an interval between 2 scorings with a GLMM. The susceptibility of the focal cow and the infectivity of her group mates were explanatory variables, and variation in exposure of susceptible cows to infectious group mates and variation in the infection pressure from the environment were accounted for in the offset. For this model no associations were observed between SNP and host susceptibility or host infectivity.

The GLMM was founded in epidemiological theory, assuming a susceptible-infected-susceptible model with an environmental route via which cows were infected. In contrast to the linear model, this model accounts for variation in the exposure of susceptible cows to pathogens spread by their infectious herd mates into the environment. Moreover, the GLMM agrees better with the non-Gaussian distribution of the y-variable. Hence, despite the absence of suggestive associations between SNP and the trait, we expect that the results of the GLMM may be more reliable.

The total infectious pressure coming from the environment was composed of claws that were infectious at the start of an interval, and of the contributions of infectious claws of cows that were infectious at an earlier stage. In the offset we accounted for the total infectious pressure from all previous infections observed during the entire experiment. Thus, with this offset, susceptibility estimates were corrected for the total variation in infectious pressure. Infectivity estimates, however, were solely based on claws that were infectious at the start of the interval; the genotypes of the cows that were infectious at an earlier stage were not considered. Not considering those genotypes reduces the power to estimate infectivity effects, especially because the survival

Table 8. Estimated heritabilities for the disease status traits analyzed with the linear model

\begin{tabular}{lcc}
\hline Trait $^{1}$ & $\sigma_{A}^{22}(\mathrm{SE})$ & $\mathrm{h}^{2}(\mathrm{SE})$ \\
\hline Infection status $^{3}$ & $0.2073(0.0316)$ & $0.28(0.01)$ \\
Active lesions observed $_{\text {Fraction }}$ active & $0.0368(0.0107)$ & $0.18(0.05)$ \\
Fraction $_{\mathrm{M} 0}$ & $0.0039(0.0014)$ & $0.13(0.05)$ \\
Fraction $_{\mathrm{M} 1}$ & $0.0516(0.0088)$ & $0.34(0.05)$ \\
Fraction $_{\mathrm{M} 2}$ & $0.0002(0.0001)$ & $0.09(0.04)$ \\
Fraction $_{\mathrm{M} 3}$ & $0.0020(0.0009)$ & $0.10(0.04)$ \\
Fraction $_{\mathrm{M} 4}$ & $0.0006(0.0002)$ & $0.16(0.05)$ \\
Fraction $_{\mathrm{M} 4.1}$ & $0.0446(0.0073)$ & $0.35(0.05)$ \\
Fraction $_{\text {free }}$ & $0.0000(0.0001)$ & $0.01(0.03)$ \\
Fraction $_{\text {F }}$ & $0.0532(0.0085)$ & $0.37(0.05)$ \\
\hline
\end{tabular}

${ }^{1}$ Fraction $_{\text {active }}=$ fraction of scorings in which a cow had at least one active lesion; fraction free $_{\text {f }}$ fraction of observations in which a cow was digital dermatitis free. Other fractions: skin where a lesion is macroscopically absent (M0), a small lesion of $0-2 \mathrm{~cm}$ (M1), a lesion of $>2$ cm (M2), a lesion covered by a scab (M3), altered skin with dyskeratosis or surface proliferation (M4), and a small lesion in addition to altered skin (M4.1).

${ }^{2}$ Additive genetic variance.

${ }^{3}$ Averaged over 75,904 analyses. 
of infectious material in the environment was long. In a previous study on the same data, we estimated that $90 \%$ of infection pressure survives from one scoring moment to the next (Biemans et al., 2018). As a consequence, $90 \%$ of the infectious pressure in each interval originated from claws that were infectious at an earlier stage. The power to find associations between SNP and infectivity should increase when the claws of all cows that contribute to the infection pressure are considered. However, in the statistical software we did not manage to keep track of all the weighted infectivity genotypes. Future GLMM on DD transmission should be extended to incorporate the weighted contribution of these claws to infectivity as well.

\section{Model Comparison}

We analyzed DD-related host traits with a linear model and with a GLMM. Heritability estimates for traits analyzed with the linear model ranged from 0.09 $\left(\right.$ fraction $\left._{\mathrm{m} 1}\right)$ to 0.37 (fraction $\left._{\text {free }}\right)$. These estimates are similar to slightly higher than the heritabilities for DD from previous studies (0.04 to 0.29 , depending on the model used; van der Waaij et al., 2005; van der Spek et al., 2013; Schöpke et al., 2015). We could only estimate heritabilities for the traits analyzed with the linear model; for traits analyzed with a GLMM heritability estimation is not trivial because of the link function. In our model we used a complementary log-log link function; therefore, the estimated genetic variance components are on a log scale. Back transforming these estimates to the original scale is possible [e.g., it has been done for GLMM with a logit or a probit link function (Dempster and Lerner, 1950; Roehe and Kalm, 2000)], but the transformation for a complementary log-log link function needs to be derived before it can be applied to the estimates in this study.

For some traits estimated with the linear model, there was a moderate correlation $(\sim 0.70)$ with SNP effects estimated with the GLMM; this correlation was smaller than the correlations between SNP effects of similar traits analyzed with the linear model. This difference suggests that for some traits (e.g., infection status and fraction $_{\mathrm{M} 4}$ ) the linear model may also have captured genetic variation in the duration of the infectious period, which is not captured by the GLMM. The ability to capture this variation might explain why we found suggestive SNP with the linear model but not with the GLMM. However, these suggestive SNP may also result from violation of model assumptions. The linear model assumed a Gaussian distribution of residuals in the analyses, which was clearly not the case. This may have caused the marginal inflation of $P$-values, 
whereas $P$-values from the GLMM showed no inflation at all (see quantile-quantile plots in the Appendix).

An advantage of the GLMM over a linear model is that the GLMM allowed us to correct susceptibility estimates for variation in exposure and to include variation in infectivity of the group mates. Anacleto et al. (2015) showed that susceptibility estimates are more accurate when genetic variation in infectivity is accounted for. Therefore, even when infectivity is not the trait of interest, it might be beneficial to include infectivity in the model to accurately estimate susceptibility (Anacleto et al., 2015). However, for infectious diseases where the pathogen survives in the environment, such as DD, the GLMM needs to be extended to better account for the full genetic variation in infectivity via the environment.

\section{CONCLUSIONS}

We used a GWAS to identify SNP associated with different DD-related host traits. With a linear model we detected 4 suggestive associations for disease status traits on 3 different chromosomes. Heritability estimates ranged from 0.09 for fraction $_{\mathrm{m} 1}$ to 0.37 for fraction $_{\text {free. With a GLMM we fitted SNP effects for }}$ host susceptibility and host infectivity, while taking the variation in exposure of the susceptible cow to infectious herd mates into account. With the GLMM we did not find any significant or suggestive associations, but SNP effects of the trait infection status of linear model had a substantial correlation (0.70) with SNP effects for susceptibility of the GLMM. We expect that the accuracy of SNP effects for susceptibility increases if the trait is analyzed with an extended GLMM that better accounts for the full genetic variation in infectivity via the environment.

\section{ACKNOWLEDGMENTS}

We thank Natasja Boots (Quantitative Veterinary Epidemiology, Wageningen University and Research, Wageningen, the Netherlands) for her help with the phenotype collection, the farmers for their participation, and breeding company CRV (Arnhem, the Netherlands) for providing the genotype data. This work, with project number 847.13.004, is part of the research program "Meer met Minder" financed by the Netherlands Organization for Scientific Research (NWO; The Hague, the Netherlands).

\section{REFERENCES}

Anacleto, O., L. A. Garcia-Cortés, D. Lipschutz-Powell, J. A. Woolliams, and A. B. Doeschl-Wilson. 2015. A novel statistical model to estimate host genetic effects affecting disease transmission. Genetics 201:871-884.
Anche, M. T., P. Bijma, and M. C. M. De Jong. 2015. Genetic analysis of infectious diseases: Estimating gene effects for susceptibility and infectivity. Genet. Sel. Evol. 47:85.

Anche, M. T., M. C. M. de Jong, and P. Bijma. 2014. On the definition and utilization of heritable variation among hosts in reproduction ratio $\mathrm{R} 0$ for infectious diseases. Heredity 113:364-374.

Argáez-Rodríguez, F. J., D. W. Hird, J. H. de Anda, D. H. Read, and A. Rodríguez-Lainz. 1997. Papillomatous digital dermatitis on a commercial dairy farm in Mexicali, Mexico: Incidence and effect on reproduction and milk production. Prev. Vet. Med. 32:275-286.

Berry, S. L., D. H. Read, T. R. Famula, A. Mongini, and D. Döpfer. 2012. Long-term observations on the dynamics of bovine digital dermatitis lesions on a California dairy after topical treatment with lincomycin HCl. Vet. J. 193:654-658.

Biemans, F., P. Bijma, N. M. Boots, and M. C. M. de Jong. 2018. Digital dermatitis in dairy cattle: The contribution of different disease classes to transmission. Epidemics 23:76-84.

Biemans, F., M. C. M. Jong, and P. Bijma. 2017. A model to estimate effects of SNPs on host susceptibility and infectivity for an endemic infectious disease. Genet. Sel. Evol. 49:53.

Bravo de Rueda, C. B., M. C. M. de Jong, P. L. Eblé, and A. Dekker. 2015. Quantification of transmission of foot-and-mouth disease virus caused by an environment contaminated with secretions and excretions from infected calves. Vet. Res. 46:43.

Bruijnis, M. R., B. Beerda, H. Hogeveen, and E. N. Stassen. 2012. Assessing the welfare impact of foot disorders in dairy cattle by a modeling approach. Animal 6:962-970.

Bruijnis, M. R., H. Hogeveen, and E. Stassen. 2010. Assessing economic consequences of foot disorders in dairy cattle using a dynamic stochastic simulation model. J. Dairy Sci. 93:2419-2432.

Calus, M. P. L., and J. Vandenplas. 2016. Calc_grm - A program to compute pedigree, genomic, and combined relationship matrices. ABGC, Wageningen UR Livestock Research, Wageningen, the Netherlands.

Demirkan, I., R. L. Walker, R. D. Murray, R. W. Blowey, and S. D. Carter. 1999. Serological evidence of spirochaetal infections associated with digital dermatitis in dairy cattle. Vet. J. 157:69-77.

Dempster, E. R., and I. M. Lerner. 1950. Heritability of threshold characters. Genetics 35:212.

Döpfer, D. 2009. Digital dermatitis: The dynamics of digital dermatitis in dairy cattle and the manageable state of disease. In Proc. CanWest Veterinary Conference, Banff AB, Canada. http://www hoofhealth.ca/Dopfer.pdf.

Döpfer, D., A. A. H. M. ter Huurne, J. L. Cornelisse, A. J. A. M. Van Asten, A. Koopmans, F. A. Meijer, Y. H. Schukken, I. Szakall, W. Klee, and R. B. Bosma. 1997. Histological and bacteriological evaluation of digital dermatitis in cattle, with special reference to spirochaetes and Campylobacter faecalis. Vet. Rec. 140:620-623.

Gilmour, A. R., B. J. Gogel, B. R. Cullis, S. J. Welham, and R. Thompson. 2015. ASReml user guide release 4.1 structural specification. VSN International Ltd., Hemel Hempstead, UK.

Holzhauer, M. 2006. Claw Health in Dairy Cows in The Netherlands: Epidemiological aspects of different claw disorders in dairy cattle in The Netherlands. Utrecht University, Utrecht, the Netherlands.

Holzhauer, M., C. Hardenberg, C. J. M. Bartels, and K. Frankena. 2006. Herd-and cow-level prevalence of digital dermatitis in the Netherlands and associated risk factors. J. Dairy Sci. 89:580-588.

Laven, R. A., and D. N. Logue. 2006. Treatment strategies for digital dermatitis for the UK. Vet. J. 171:79-88.

Lipschutz-Powell, D., J. A. Woolliams, P. Bijma, R. Pong-Wong, M. L. Bermingham, and A. B. Doeschl-Wilson. 2012. Bias, accuracy, and impact of indirect genetic effects in infectious diseases. Front. Genet. 3:215.

Lipschutz-Powell, D., J. A. Woolliams, and A. B. Doeschl-Wilson. 2014. A unifying theory for genetic epidemiological analysis of binary disease data. Genet. Sel. Evol. 46:15.

Malchiodi, F., A. Koeck, A. Christen, F. Schenkel, D. Kelton, and F. Miglior. 2015. Genetic parameters and genome wide association study of individual hoof lesions in Canadian Holsteins using different contemporary groups. 
Manske, T., J. Hultgren, and C. Bergsten. 2002. Topical treatment of digital dermatitis associated with severe heel-horn erosion in a Swedish dairy herd. Prev. Vet. Med. 53:215-231.

Naderi, S., M. Bohlouli, T. Yin, and S. König. 2018. Genomic breeding values, SNP effects and gene identification for disease traits in cow training sets. Anim. Genet. 49:178-192.

Nielsen, B. H., P. T. Thomsen, and J. T. Sørensen. 2009. A study of duration of digital dermatitis lesions after treatment in a Danish dairy herd. Acta Vet. Scand. 51:27.

Oberbauer, A. M., A. L. Danner, J. M. Belanger, T. R. Famula, and J. M. Heguy. 2016. 0329 Genome-wide association study for loci associated with digital dermatitis and pododermatitis circumscripta in Holstein cattle. J. Anim. Sci. 94(supplement5):158.

Peterson, B. G., P. Carl, K. Boudt, R. Bennett, J. Ulrich, E. Zivot, M. Lesstel, K. Balkissoon, and D. Wuertz. 2014. PerformanceAnalytics: Econometric tools for performance and risk analysis. $\mathrm{R}$ package version 1.4. 3541.

R Core Team. 2017. R: A Language and Environment for Statistical Computing. 3.4.0 ed. R Foundation for Statistical Computing, Vienna, Austria.

Read, D. H., and R. L. Walker. 1998. Papillomatous digital dermatitis (footwarts) in California dairy cattle: Clinical and gross pathologic findings. J. Vet. Diagn. Invest. 10:67-76.

Relun, A., R. Guatteo, P. Roussel, and N. Bareille. 2011. A simple method to score digital dermatitis in dairy cows in the milking parlor. J. Dairy Sci. 94:5424-5434.

Roberts, M., and H. Heesterbeek. 1993. Bluff your way in epidemic models. Trends Microbiol. 1:343-348.

Rodríguez-Lainz, A., D. W. Hird, T. E. Carpenter, and D. H. Read. 1996. Case-control study of papillomatous digital dermatitis in Southern California dairy farms. Prev. Vet. Med. 28:117-131.

Rodriguez-Lainz, A., P. Melendez-Retamal, D. W. Hird, and D. H. Read. 1998. Papillomatous digital dermatitis in Chilean dairies and evaluation of a screening method. Prev. Vet. Med. 37:197-207.

Roehe, R., and E. Kalm. 2000. Estimation of genetic and environmental risk factors associated with pre-weaning mortality in piglets using generalized linear mixed models. Anim. Sci. 70:227-240.

Scholey, R. A. 2011. Investigating host genetic susceptibility to bovine digital dermatitis. Doctoral Diss. University of Liverpool, Liverpool, UK.

Schöpke, K., A. Gomez, K. A. Dunbar, H. H. Swalve, and D. Döpfer. 2015. Investigating the genetic background of bovine digital dermatitis using improved definitions of clinical status. J. Dairy Sci.98:8164-8174.

Sogstad, A. M., T. Fjeldaas, O. Østerås, and K. P. Forshell. 2005. Prevalence of claw lesions in Norwegian dairy cattle housed in tie stalls and free stalls. Prev. Vet. Med. 70:191-209.
Speijers, M. H. M., L. G. Baird, G. A. Finney, J. McBride, D. J. Kilpatrick, D. N. Logue, and N. E. O'Connell. 2010. Effectiveness of different footbath solutions in the treatment of digital dermatitis in dairy cows. J. Dairy Sci. 93:5782-5791.

Storey, J. D., and R. Tibshirani. 2003. Statistical significance for genomewide studies. Proc. Natl. Acad. Sci. USA 100:9440-9445.

Turner, S. D. 2014. qqman: An R package for visualizing GWAS results using QQ and Manhattan plots. bioRxiv:005165.

van der Linde, C., G. de Jong, E. P. C. Koenen, and H. Eding. 2010 Claw health index for Dutch dairy cattle based on claw trimming and conformation data. J. Dairy Sci. 93:4883-4891.

van der Spek, D., J. A. M. van Arendonk, and H. Bovenhuis. 2015. Genome-wide association study for claw disorders and trimming status in dairy cattle. J. Dairy Sci. 98:1286-1295.

van der Spek, D., J. A. M. van Arendonk, A. A. A. Vallée, and H Bovenhuis. 2013. Genetic parameters for claw disorders and the effect of preselecting cows for trimming. J. Dairy Sci. 96:6070-6078.

van der Waaij, E. H., M. Holzhauer, E. Ellen, C. Kamphuis, and G. de Jong. 2005. Genetic parameters for claw disorders in Dutch dairy cattle and correlations with conformation traits. J. Dairy Sci. 88:3672-3678.

VanRaden, P. M. 2008. Efficient methods to compute genomic predictions. J. Dairy Sci. 91:4414-4423.

Velthuis, A. G. J., M. C. M. De Jong, E. M. Kamp, N. Stockhofe, and J. H. M. Verheijden. 2003. Design and analysis of an Actinobacillus pleuropneumoniae transmission experiment. Prev. Vet. Med. 60:53-68.

Vink, W. D., G. Jones, W. O. Johnson, J. Brown, I. Demirkan, S. D. Carter, and N. P. French. 2009. Diagnostic assessment without cut-offs: Application of serology for the modelling of bovine digital dermatitis infection. Prev. Vet. Med. 92:235-248

Walker, R. L., D. H. Read, K. J. Loretz, and R. W. Nordhausen. 1995. Spirochetes isolated from dairy cattle with papillomatous digital dermatitis and interdigital dermatitis. Vet. Microbiol. 47:343-355.

Wells, S. J., L. P. Garber, and B. A. Wagner. 1999. Papillomatous digital dermatitis and associated risk factors in US dairy herds. Prev. Vet. Med. 38:11-24.

Yang, J., S. H. Lee, M. E. Goddard, and P. M. Visscher. 2011. GCTA A tool for genome-wide complex trait analysis. Am. J. Hum. Genet. 88:76-82.

Zinicola, M., F. Lima, S. Lima, V. Machado, M. Gomez, D. Döpfer, C. Guard, and R. Bicalho. 2015. Altered microbiomes in bovine digital dermatitis lesions, and the gut as a pathogen reservoir. PLoS One 10:e0120504. 


\section{APPENDIX}
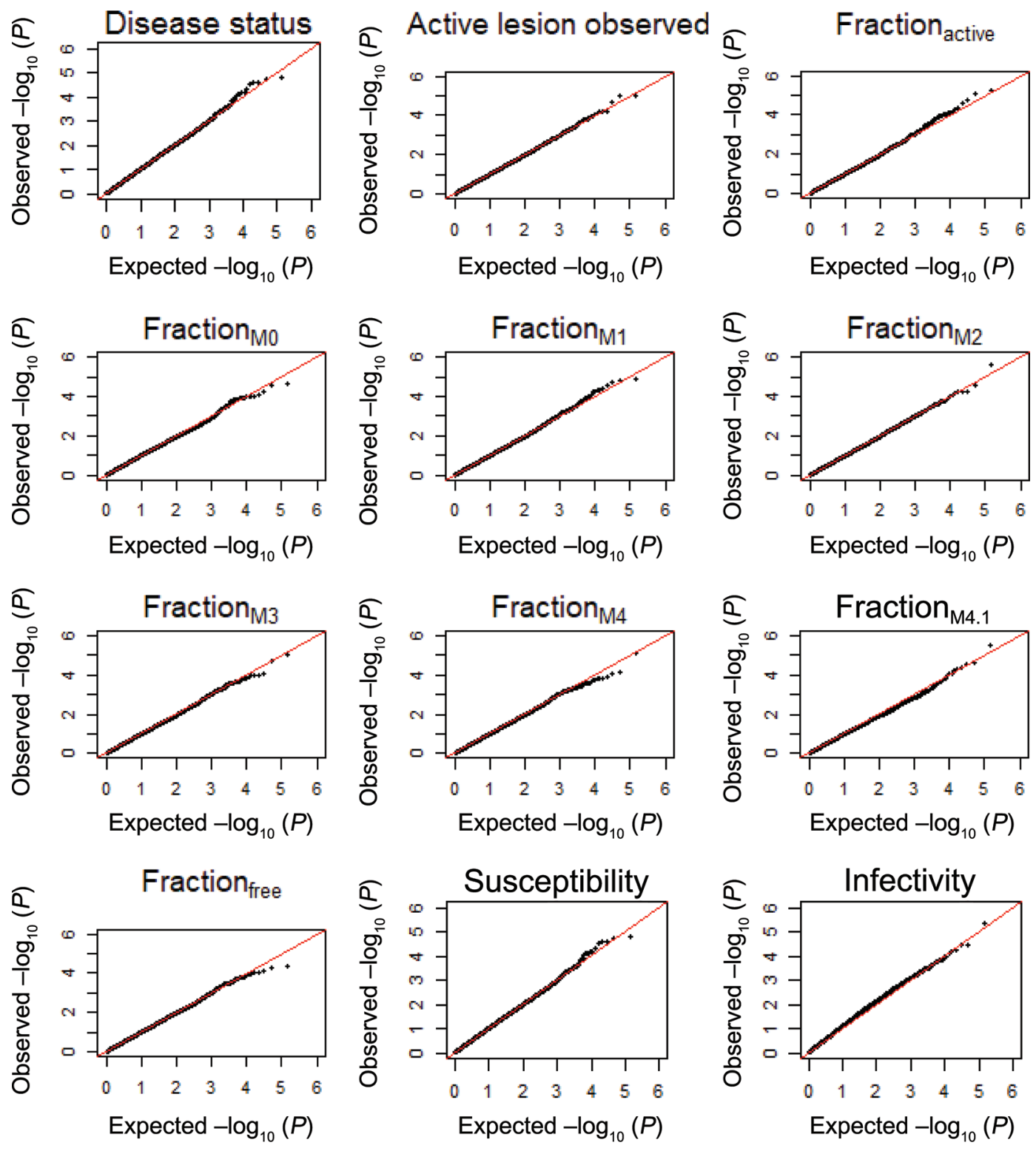

Figure A1. Quantile-quantile plots for the $P$-values from the genome-wide association studies for the traits presented in the main text

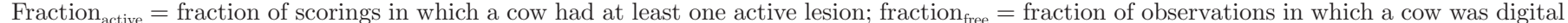
dermatitis free. Other fractions: skin where a lesion is macroscopically absent (M0), a small lesion of 0 to $2 \mathrm{~cm}$ (M1), a lesion of $>2 \mathrm{~cm}$ (M2), a lesion covered by a scab (M3), altered skin with dyskeratosis or surface proliferation (M4), and a small lesion in addition to altered skin (M4.1). 